\title{
Neck stiffness and papilloedema due to Harada syndrome
}

\author{
B M T P Nawasiwatte ${ }^{1}$, R Premaratna ${ }^{1}$, B Amarasinghe ${ }^{2}$, H J de Silva ${ }^{1}$
}

Ceylon Medical Journal 2012; 57: 88-89

\section{Case report}

A 41-year old previously healthy woman presented with severe headache, vomiting, low grade fever, bilateral eye pain, photophobia and deteriorating vision over one week. Examination showed neck stiffness and bilateral papilloedema [Figures 1 and 2]. She had no evidence of autoimmune disorders. Contrast CT brain and routine investigations were normal. CSF showed pleocytosis with negative microbiological tests. Worsening visual acuity in the absence of meningitis or an intracranial space occupying lesion prompted an urgent ophthalmological assessment which showed bilateral disc oedema with multiple exudative detachments of the sensory retina with vitreal cells, suggestive of posterior uveitis. This led to the clinical diagnosis of an incomplete form of Harada syndrome, as she had no auditory or cutaneous signs [1]. She had an excellent response to intravenous methylprednisolone followed by oral steroids. Serial visual and fundoscopy assessments showed gradual improvement in visual acuity, disc oedema and retinal detachments, and visual acuity became normal after one month of treatment [Figures 3 and 4]. Further supporting our diagnosis, four months later she presented with significant hair loss, but without any poliosis or vitiligo (integumentary signs).

Vogt-Koyanagi-Harada (VKH) syndrome is a rare inflammatory granulomatous disorder involving melanocyte containing organs causing bilateral panuveitis and retinitis (ophthalmic), meningismus and cerebrospinal fluid pleocytosis (neurological), tinnitus (auditory) and alopecia, poliosis, and vitiligo (integumentary) manifestations [1]. It has HLA-DR4, HLADR53, and HLA-DQ4 associations [2]. The disease commonly affects dark skinned people, women more than men, of any age group. Although an infectious aetiology is often postulated, autoimmune origins have also been considered as VKH is associated with certain autoimmune disorders such as type I diabetes mellitus, Hashimoto's thyroiditis, polyglandular syndrome and IgA nephropathy $(3,4,5)$. Exclusion of a history of ocular trauma, surgery or

${ }^{1}$ University Medical Unit and ${ }^{2}$ Department of Opthalmology, Colombo North Teaching Hospital, Ragama, Sri Lanka. Correspondence: BMTPN, e-mail: <thusha.nawasiwatte@gmail.com>. Received 21 April 2011 and revised version accepted 5 January 2012. Competing interests: none declared. 

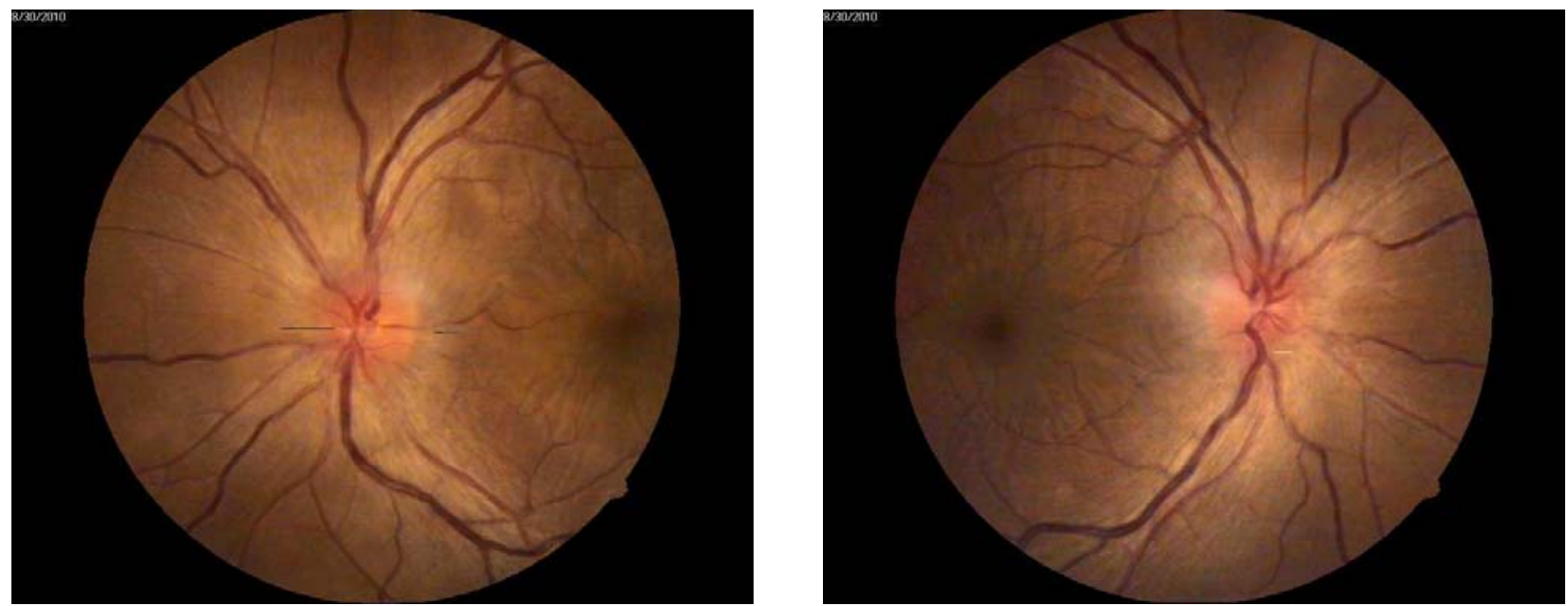

Figures 1 and 2. Fundoscopic appearance of right and left retina during the acute presentation showing papilloedema and peri-macular oedema.
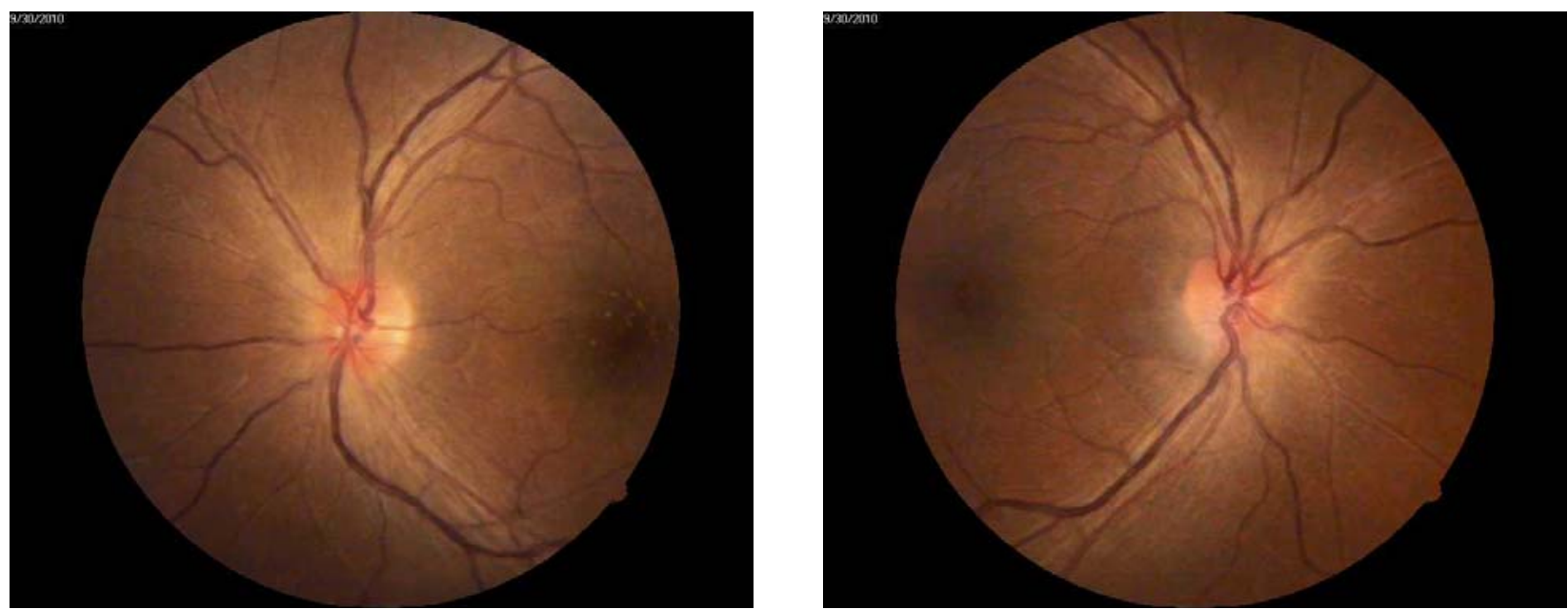

Figure 3 and 4: Fundoscopy of both eyes showing improvement of retinal abnormalities after one month of steroid treatment.

other ocular diseases is needed for the diagnosis of VKH. There are 3 categories: patients with complete VKH have visual, neurologic and auditory abnormalities together with integumentary signs; in incomplete VKH the mandatory eye signs are coupled either with neurologic plus auditory manifestations without integumentary signs or vice versa; probable VKH includes those with isolated ocular disease.

Reversible and irreversible vision loss, glaucoma, and cataract can occur due to VKH syndrome, and the final visual outcome depends on the rapidity of treatment. Skin changes are usually permanent, but hearing is restored completely in most patients. We wish to highlight the importance of a prompt ophthalmological assessment in similar patients.

\section{Acknowledgments}

We thank Mr. D R Fonseka, for the retinal photography.

\section{References}

1. Walton RC. Vogt-Koyanagi-Harada's disease. International Committee on Nomenclature revised criteria for the diagnosis of VKH disease in e-medicine (http://emedicine.medscape. com/article/1229432-overview)

2. Andreoli CM, Foster CS. Vogt-Koyanagi-Harada disease. International Ophthalmology Clinics 2006; 46: 111-22.

3. Sunakawa M, Okinami S. Epstein-Barr virus-related antibody pattern in uveitis. Japanese Journal of Ophthalmology 1985; 29: 423-8.

4. Yamaki K, Gocho K, Hayakawa K, Kondo I, Sakuragi S. Tyrosinase family proteins are antigens specific to VogtKoyanagi-Harada disease. Journal of Immunology 2000; 165: 7323-9.

5. Al Hemidan AI, Tabbara KF, Althomali T. Vogt-KoyanagiHarada associated with diabetes mellitus and celiac disease in a 3-year old girl. European Journal of Ophthalmology 2006; 16: 173-7. 